\title{
ON THE MAIN EIGENVALUES OF UNIVERSAL ADJACENCY MATRICES AND U-CONTROLLABLE GRAPHS*
}

\author{
ALEXANDER FARRUGIA $^{\dagger}$ AND IRENE SCIRIHA $^{\dagger}$
}

\begin{abstract}
A universal adjacency matrix $\mathbf{U}$ of a graph $G$ is a linear combination of the $0-1$ adjacency matrix $\mathbf{A}$, the diagonal matrix of vertex degrees $\mathbf{D}$, the identity matrix $\mathbf{I}$ and the matrix $\mathbf{J}$ each of whose entries is 1 . A main eigenvalue of $\mathbf{U}$ is an eigenvalue having an eigenvector that is not orthogonal to the all-ones vector. It is shown that the number of distinct main eigenvalues of $\mathbf{U}$ associated with a simple graph $G$ is at most the number of orbits of any automorphism of $G$. The definition of a $\mathbf{U}$-controllable graph is given using control-theoretic techniques and several necessary and sufficient conditions for a graph to be $\mathbf{U}$-controllable are determined. It is then demonstrated that $\mathbf{U}$-controllable graphs are asymmetric and that the converse is false, showing that there exist both regular and non-regular asymmetric graphs that are not $\mathbf{U}$-controllable for any universal adjacency matrix $\mathbf{U}$. To aid in the discovery of these counterexamples, a $\gamma$-Laplacian matrix $\mathcal{L}(\gamma)$ is used, which is a simplified form of $\mathbf{U}$. It is proved that any $\mathbf{U}$-controllable graph is a $\mathcal{L}(\gamma)$-controllable graph for some parameter $\gamma$.
\end{abstract}

Key words. Main eigenvalue, Universal adjacency matrix, Asymmetric graph, $\gamma$-Laplacian matrix, U-Controllable graph.

AMS subject classifications. 05C50, 15A18, 93B05.

1. Preliminaries. Only simple graphs will be considered in this paper, that is, undirected graphs with no loops, no multiple edges, and no weighted edges. The sets of vertices and edges of a graph $G$ will be denoted by $\mathcal{V}(G)$ and $\mathcal{E}(G)$ respectively, with $n=|\mathcal{V}(G)|$. The adjacency matrix $\mathbf{A}$ of a graph $G$ is the $n \times n$ matrix $\left(a_{i j}\right)$ where $a_{i j}=1$ if $\{i, j\} \in \mathcal{E}(G)$ and is 0 otherwise. If $v_{1}, \ldots, v_{n}$ are the vertices of $G$, then the matrix $\mathbf{D}$ is the diagonal matrix whose $i^{\text {th }}$ diagonal entry is the degree of $v_{i}$ for all $1 \leq i \leq n$. The vector $\mathbf{j}$ denotes the vector $\left(\begin{array}{llll}1 & 1 & \cdots & 1\end{array}\right)^{\top}$ of all ones. We shall denote the identity matrix by $\mathbf{I}$ and the square matrix of all ones by $\mathbf{J}$.

A graph $G$ is said to be regular of degree $\rho$ if $\mathbf{D}=\rho \mathbf{I}$, otherwise, $G$ is non-regular. If $G$ is any graph, then the cone $G_{\triangleright}$ of $G$ is obtained from the graph $G$ by inserting a vertex adjacent to all the vertices of $G$. The complement of $G$, denoted by $\bar{G}$, is the graph with $\mathcal{V}(\bar{G})=\mathcal{V}(G)$ where two distinct vertices are adjacent if and only if they are not adjacent in $G$.

*Received by the editors on March 10, 2015. Accepted for publication on October 29, 2015. Handling Editor: Bryan L. Shader.

${ }^{\dagger}$ Department of Mathematics, University of Malta, Malta (alex.farrugia@um.edu.mt, isci1@um.edu.mt). 
An automorphism of a graph $G$ is a mapping $\sigma$ from $\mathcal{V}(G)$ to itself preserving adjacencies, that is, $\{i, j\} \in \mathcal{E}(G)$ if and only if $\{\sigma(i), \sigma(j)\} \in \mathcal{E}(G)$. The set of all automorphisms of $G$ forms the automorphism group of $G$ with the operation of composition. For any automorphism $\sigma$, we associate the permutation matrix $\mathbf{P}_{\sigma}$ such that the $i^{\text {th }}$ column of $\mathbf{P}_{\sigma}$ is the $j^{\text {th }}$ column of $\mathbf{I}$ if and only if $\sigma(i)=j$. Any automorphism $\sigma$ can be expressed as a product of disjoint cycles, and this determines a partition of $\mathcal{V}(G)$ into orbits. If $G$ has a trivial automorphism group consisting only of the identity automorphism, then $G$ is said to be asymmetric.

Let $\mathbf{M}$ be a real and symmetric matrix satisfying the equation $\mathbf{M x}=\lambda \mathbf{x}$ for some scalar $\lambda$ and some nonzero vector $\mathbf{x}$. The vector $\mathbf{x}$ is an eigenvector associated with the eigenvalue $\lambda$ of $\mathbf{M}$. The set of all eigenvectors associated with $\lambda$, together with the zero vector, form a vector space, called the eigenspace of $\lambda$. The spectrum of $\mathbf{M}$ is the multiset of all eigenvalues of $\mathbf{M}$, that is, the multiset of all solutions of the characteristic equation $\operatorname{det}(\lambda \mathbf{I}-\mathbf{M})=0$. If 0 is an eigenvalue of $\mathbf{M}$, then $\mathbf{M}$ is said to be singular, in which case the nullspace $\operatorname{ker}(\mathbf{M}) \neq\{\mathbf{0}\}$. Since $\mathbf{M}$ is real and symmetric, the multiplicity of an eigenvalue $\lambda$ is the dimension of its eigenspace, or, equivalently, the number of times it is repeated in the spectrum of $\mathbf{M}$. An eigenvalue is said to be simple if its multiplicity is one. A main eigenvalue of $\mathbf{M}$ is an eigenvalue having some associated eigenvector not orthogonal to $\mathbf{j}$, referred to as a main eigenvector. On the other hand, the eigenspace of a non-main eigenvalue contains only non-main eigenvectors, that is, eigenvectors that are orthogonal to $\mathbf{j}$.

In Section 2, we describe the universal adjacency matrix $\mathbf{U}[1,8]$ associated with a graph $G$ and prove that its main eigenvalues must have an associated eigenvector of a certain form, from which an upper bound on the number of main eigenvalues of $\mathbf{U}$ is deduced. In Section 3, we summarize the principal results from control theory that will be used in subsequent sections, leading to the definition of a $\mathbf{U}$-controllable graph $G$ in Section 4, where several characterisations of such a graph are presented. We prove in Section 5 that $\mathbf{U}$-controllable graphs are asymmetric; yet, there exist regular, asymmetric graphs $G$ that are not $\mathbf{U}$-controllable. In Section 6, we introduce the $\gamma$-Laplacian matrix $\mathcal{L}(\gamma)$, which is a simpler form of the matrix $\mathbf{U}$, and show that a graph is $\mathbf{U}$-controllable if and only if it is $\mathcal{L}(\gamma)$-controllable for an appropriate value of the scalar $\gamma$. This facilitates our search for non-regular asymmetric graphs that are not $\mathbf{U}$-controllable, and indeed, one such graph is presented in Section 7 .

2. The universal adjacency matrix U. The reference [8] (see also [1]) defined a universal adjacency matrix associated with a simple graph $G$ to be a matrix of the form

$$
\mathbf{U}=\gamma_{A} \mathbf{A}+\gamma_{D} \mathbf{D}+\gamma_{I} \mathbf{I}+\gamma_{J} \mathbf{J}, \quad \gamma_{A} \neq 0
$$


for some nonzero scalar $\gamma_{A}$ and some arbitrary scalars $\gamma_{I}, \gamma_{D}$ and $\gamma_{J}$. The authors of [8] determined the graphs that admit a universal adjacency matrix having only two distinct eigenvalues. In [1], the minimum rank of $\mathbf{U}$ was studied. In this article, we consider the number of distinct main eigenvalues of $\mathbf{U}$ and relate this number to what we call $\mathbf{U}$-controllable graphs.

The matrix $\mathbf{U}$ includes five of the most commonly-used matrices in algebraic graph theory, namely:

- the adjacency matrix $\mathbf{A}$ of $G\left(\gamma_{A}=1, \gamma_{I}=\gamma_{D}=\gamma_{J}=0\right)$;

- the Laplacian matrix $\mathbf{L}=\mathbf{D}-\mathbf{A}$ of $G\left(\gamma_{A}=-1, \gamma_{D}=1, \gamma_{I}=\gamma_{J}=0\right)$;

- the signless Laplacian matrix $\mathbf{Q}=\mathbf{D}+\mathbf{A}$ of $G\left(\gamma_{A}=1, \gamma_{D}=1, \gamma_{I}=\gamma_{J}=0\right)$;

- the adjacency matrix $\overline{\mathbf{A}}=\mathbf{J}-\mathbf{I}-\mathbf{A}$ of $\bar{G}\left(\gamma_{A}=-1, \gamma_{I}=-1, \gamma_{J}=1, \gamma_{D}=0\right)$;

- the Seidel matrix $\mathcal{S}=\mathbf{J}-\mathbf{I}-2 \mathbf{A}$ of $G\left(\gamma_{A}=-2, \gamma_{I}=-1, \gamma_{J}=1, \gamma_{D}=0\right)$.

Thus, $\mathbf{U}$ allows us to consider all the above five matrices (and more) collectively in order to obtain results that would be valid for all of them at once. Indeed, the following theorem generalizes the result found in $[9,10]$, there shown to be true only for the adjacency matrix $\mathbf{A}$, to the case of a universal adjacency matrix $\mathbf{U}$.

TheOrem 2.1. [6, Theorem 5.4] If $\mathbf{P}$ is an arbitrary permutation matrix and $G$ is a graph with associated universal adjacency matrix $\mathbf{U}$, then $\mathbf{P}$ is the permutation matrix $\mathbf{P}_{\sigma}$ of an automorphism $\sigma$ of $G$ if and only if $\mathbf{P U}=\mathbf{U P}$.

A consequence to the above theorem is the result below.

THEOREM 2.2. Let $\mathbf{U}$ be a universal adjacency matrix for a graph $G, \lambda$ be a main eigenvalue of $\mathbf{U}$ and $\sigma$ be an automorphism of $G$. Then there is a main eigenvector $\mathbf{x}$ associated with $\lambda$ such that $\mathbf{P}_{\sigma} \mathbf{x}=\mathbf{x}$.

Proof. Let $\mathbf{U} \mathbf{y}=\lambda \mathbf{y}$ such that $\mathbf{j}^{\top} \mathbf{y} \neq 0$. If $\mathbf{P}_{\sigma} \mathbf{y}=\mathbf{y}$, then we are done. If $\mathbf{P}_{\sigma} \mathbf{y} \neq$ $\mathbf{y}$, then let $r$ be the order of $\mathbf{P}_{\sigma}$ and consider the vector $\mathbf{x}=\left(\mathbf{I}+\mathbf{P}_{\sigma}+\mathbf{P}_{\sigma}^{2}+\cdots+\mathbf{P}_{\sigma}^{r-1}\right) \mathbf{y}$. By Theorem 2.1, we have

$$
\begin{aligned}
\mathbf{U x} & =\mathbf{U}\left(\mathbf{I}+\mathbf{P}_{\sigma}+\mathbf{P}_{\sigma}^{2}+\cdots+\mathbf{P}_{\sigma}^{r-1}\right) \mathbf{y}=\left(\mathbf{I}+\mathbf{P}_{\sigma}+\mathbf{P}_{\sigma}^{2}+\cdots+\mathbf{P}_{\sigma}^{r-1}\right) \mathbf{U} \mathbf{y} \\
& =\lambda\left(\mathbf{I}+\mathbf{P}_{\sigma}+\mathbf{P}_{\sigma}^{2}+\cdots+\mathbf{P}_{\sigma}^{r-1}\right) \mathbf{y}=\lambda \mathbf{x} .
\end{aligned}
$$

Also, note that $\mathbf{j}^{\top} \mathbf{x}=\mathbf{j}^{\top}\left(\mathbf{I}+\mathbf{P}_{\sigma}+\mathbf{P}_{\sigma}^{2}+\cdots+\mathbf{P}_{\sigma}^{r-1}\right) \mathbf{y}=r \mathbf{j}^{\top} \mathbf{y} \neq 0$. Therefore, $\mathbf{x} \neq \mathbf{0}$, and thus, $\mathbf{x}$ is an eigenvector in the eigenspace of $\lambda$.

Moreover,

$$
\begin{aligned}
\mathbf{P}_{\sigma} \mathbf{x}=\mathbf{P}_{\sigma}\left(\mathbf{I}+\mathbf{P}_{\sigma}+\cdots+\mathbf{P}_{\sigma}^{r-1}\right) \mathbf{y} & =\left(\mathbf{P}_{\sigma}+\mathbf{P}_{\sigma}^{2}+\cdots+\mathbf{P}_{\sigma}^{r}\right) \mathbf{y} \\
& =\left(\mathbf{I}+\mathbf{P}_{\sigma}+\cdots+\mathbf{P}_{\sigma}^{r-1}\right) \mathbf{y}=\mathbf{x}
\end{aligned}
$$

as required. 
We remark that the analogous result for non-main eigenvalues is false in general. The three statements $\mathbf{U} \mathbf{x}=\lambda \mathbf{x}, \mathbf{j}^{\top} \mathbf{x}=0$ and $\mathbf{P}_{\sigma} \mathbf{x}=\mathbf{x}$ would not be enough to prove that $\mathbf{x}$ is an eigenvector of a non-main eigenvalue $\lambda$ with the requirements of Theorem 2.2 , as $\mathbf{x}$ could possibly be the zero vector. This happens, for example, if we take $\mathbf{y}$ to be the eigenvector of $\lambda=-1$ of the matrix $\mathbf{A}$ in Table 2.1 (in page 817).

Moreover, note that the matrix $\mathbf{M}=\mathbf{I}+\mathbf{P}_{\sigma}+\mathbf{P}_{\sigma}^{2}+\cdots+\mathbf{P}_{\sigma}^{r-1}$ used in the proof of Theorem 2.2 is singular if and only if $r>1$. Indeed, if $r=1$, then $\mathbf{M}=\mathbf{I}$, whilst if $r>1$, then $\mathbf{M}\left(\mathbf{I}-\mathbf{P}_{\sigma}\right)=\mathbf{I}-\mathbf{P}_{\sigma}^{r}=\mathbf{0}$. Thus, when $r>1$, the matrices $\mathbf{M}$ and $\left(\mathbf{I}-\mathbf{P}_{\sigma}\right)$ are nonzero matrix divisors of the zero matrix, so that the nonzero columns of $\mathbf{M}$ are in the nullspace of the matrix $\left(\mathbf{I}-\mathbf{P}_{\sigma}\right)$.

Theorem 2.2 can be restated in the following way.

COROLlary 2.3. If $\lambda$ is a main eigenvalue of a universal adjacency matrix $\mathbf{U}$ associated with a graph $G$ and $\sigma$ is an automorphism of $G$, then there exists an eigenvector $\mathbf{x}$ of $\lambda$ whose entries do not sum to zero. Moreover, the entries of $\mathbf{x}$ are constant on each orbit of vertices determined by $\sigma$.

Note that Theorem 2.2 and Corollary 2.3 hold for any main eigenvalue of a universal adjacency matrix $\mathbf{U}$, regardless of its multiplicity.

We now use Corollary 2.3 to show that the number of main eigenvalues of a universal adjacency matrix $\mathbf{U}$ associated with a graph $G$ is limited to at most the number of orbits of any automorphism of $G$. This is a generalisation of the result in [3, Theorem 4], there proved only for the adjacency matrix $\mathbf{A}$ associated with $G$.

THEOREM 2.4. The number of orbits of any automorphism of a graph $G$ is an upper bound for the number of distinct main eigenvalues of any universal adjacency matrix $\mathbf{U}$ associated with $G$.

Proof. Let $\sigma$ be an automorphism of $G$ expressed as the product of $q$ cycles of length $r_{1}, r_{2}, \ldots, r_{q}$ respectively. By Corollary 2.3, each distinct main eigenvalue of $\mathbf{U}$ has an associated eigenvector of the form

$$
(\overbrace{x_{1} x_{1} \cdots x_{1}}^{r_{1}} \overbrace{x_{2} x_{2} \cdots x_{2}}^{r_{2}} \cdots \overbrace{x_{q} x_{q} \cdots x_{q}}^{r_{q}})^{\top},
$$

after possibly reordering the vertices of $G$. Since these eigenvectors are linearly independent, the number of distinct main eigenvalues of $\mathbf{U}$ cannot exceed $q$.

We demonstrate these results for the graph $G$ in Figure 2.1. The permutation $\sigma=\left(\begin{array}{lll}1 & 2 & 3\end{array}\right)\left(\begin{array}{ll}4 & 5\end{array}\right)$ is an automorphism of $G$, showing that there is a partition of the vertices into four orbits. The eigenspaces of $\mathbf{A}, \mathbf{L}, \mathbf{Q}, \overline{\mathbf{A}}$ and $\mathcal{S}$ are displayed in Table 2.1. The eigenvalues in boldface are main, and we observe that each of the matrices has at most four main eigenvalues, as expected from Theorem 2.4. In addition, 
note that each main eigenvalue has one eigenvector $\mathbf{x}=\left(\begin{array}{llll}x_{1} & x_{2} & \cdots & x_{n}\end{array}\right)^{\top}$ where $x_{1}=x_{2}=x_{3}$ and $x_{4}=x_{5}$, conforming with the automorphism $\sigma$. Moreover, note that the converse of Theorem 2.2 is false, since there are non-main eigenvalues, notably the eigenvalues $5.262,3.340$ and 0.398 of $\mathbf{L}$, with all their eigenvectors satisfying these equalities as well.

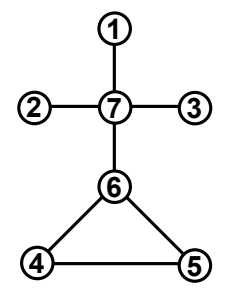

FIG. 2.1. An example graph.

We remark that any two universal adjacency matrices having the same coefficients of $\mathbf{A}$ and $\mathbf{D}$ must have the same number of distinct main eigenvalues, since they share the same non-main eigenvectors. On the other hand, two universal adjacency matrices with different coefficients of $\mathbf{A}$ or $\mathbf{D}$ can have a different number of distinct main eigenvalues. This becomes evident when considering $\mathbf{U}$-controllable graphs in Section 4.

3. Control theory. Before we introduce $\mathbf{U}$-controllable graphs, we summarize various results from control theory, on which the definition of $\mathbf{U}$-controllable graphs is based. These results are also mentioned in [6].

A networked dynamical system is a set of $n$ agents and a set of links interconnecting these agents. Such a networked dynamical system can thus be represented as a graph $G$, with the $n$ vertices denoting the agents and the edges denoting the interconnections between distinct pairs of agents. Each edge is weighted according to the strength of the exchange link between the two agents, giving rise to a weighted graph. However, in this article we assume that every exchange link between any two interconnected agents has the same strength, which is why only simple graphs are considered.

The state of each agent is affected by the information exchanged with other agents through the interconnections, and also by signals received from external leaders that attempt to control these agents in order to direct their information to some predetermined state. The number of leaders in a networked dynamical system could be arbitrary, however, in this paper we shall assume that exactly one leader agent is present. Thus, we end up with a follower graph, which is the original graph $G$ of $n$ follower agents, and a system graph which is the graph $G$ together with one leader 


\begin{tabular}{|c|c|c|c|c|}
\hline Eigenvalues of $\mathrm{A}$ & \multicolumn{4}{|c|}{ Eigenbases } \\
\hline 2.369 & $\begin{array}{|lll|}0.422 & 0.422 & 0.422 \\
\end{array}$ & 0.8050 .805 & $1.102 \quad 1$ & ]) ${ }^{\top}$ \\
\hline 1.527 & \begin{tabular}{|llllll}
0.655 & 0.655 & 0.655 & -
\end{tabular} & $-0.835-0.835$ & \begin{tabular}{|l|l|}
-0.440 \\
\end{tabular} & \begin{tabular}{l|l}
1 & $\mathrm{~T}$ \\
\end{tabular} \\
\hline 0 & \multicolumn{4}{|c|}{$\begin{array}{lllllll}\left(\begin{array}{cccccccc}-1 & 0 & 1 & 0 & 0 & 0 & 0 \\
-1 & -1 & 1 & 0 & 0 & 0 & 0 & 0\end{array}\right)^{\top} \\
\end{array}$} \\
\hline-0.789 & $-1.270-1.270-1.270$ & $0-1.690-1.69$ & 3.021 & $1)^{\top}$ \\
\hline-1 & \multicolumn{4}{|c|}{$\begin{array}{lllllll} & \left(\begin{array}{llllll}0 & 0 & 0 & -1 & 1 & 0\end{array}\right)^{\top} \\
\end{array}$} \\
\hline-2.107 & $\left(\begin{array}{lll}-0.475 & -0.475 & -0.475\end{array}\right.$ & $\begin{array}{lll}75 & 0.220 & 0.220 \\
\end{array}$ & -0.683 & 1 (1) $)^{\top}$ \\
\hline
\end{tabular}

\begin{tabular}{|c|c|}
\hline Eigenvalues of $\mathrm{L}$ & Eigenbases \\
\hline 5.262 & $\left.\begin{array}{lllllll}(-0.235 & -0.235 & -0.235 & 0.131 & 0.131 & -0.558 & 1\end{array}\right)^{\top}$ \\
\hline 3.340 & $\left.\begin{array}{lllllll}-0.427 & -0.427 & -0.427 & -0.830 & -0.830 & 1.942 & 1\end{array}\right)^{\top}$ \\
\hline 3 & $\left(\begin{array}{lllllll}0 & 0 & 0 & -1 & 1 & 0 & 0\end{array}\right)^{1}$ \\
\hline 1 & 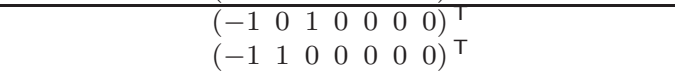 \\
\hline 0.398 & $\left(\begin{array}{lllllll}1.662 & 1.662 & 1.662 & -2.301 & -2.301 & -1.384 & 1\end{array}\right)^{\top}$ \\
\hline 0 & 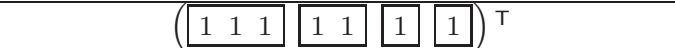 \\
\hline
\end{tabular}

\begin{tabular}{|c|c|c|c|c|}
\hline Eigenvalues of $\mathrm{Q}$ & \multicolumn{4}{|c|}{ Eigenbases } \\
\hline 5.354 & $\begin{array}{|lll|}0.230 & 0.230 & 0.230 \\
\end{array}$ & \begin{tabular}{|lll}
0.282 & 0.282 \\
\end{tabular} & 0.665 & 1 (1) $)^{\top}$ \\
\hline 4 & $\begin{array}{|lll|}1 & 1 & 1\end{array}$ & - & 3 (3) $)^{\mathrm{T}}$ & \\
\hline 1.524 & $\begin{array}{|lll|}1.910 & 1.910 & 1.910 \\
\end{array}$ & $\begin{array}{|ll|}5.558 & 5.558 \\
\end{array}$ & -8.207 & \begin{tabular}{|l|l}
1 & $\mathrm{~T}$ \\
\end{tabular} \\
\hline 1 & 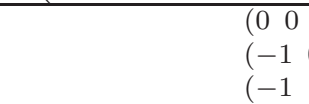 & $\left.\begin{array}{ccccccc} & -1 & -1 & 1 & 0 & 0 \\
0 & 1 & 0 & 0 & 0 & T^{\top} \\
1 & 0 & 0 & 0 & 0 & 0 & 0\end{array}\right)^{\top}$ & & \\
\hline 0.123 & $(-1.140-1.140-1.14$ & 0 & -0.45 & \begin{tabular}{l|l|l}
8 & 1 & ${ }^{\top}$
\end{tabular} \\
\hline
\end{tabular}

\begin{tabular}{|c|c|c|c|c|}
\hline Eigenvalues of $\overline{\mathrm{A}}$ & \multicolumn{4}{|c|}{ Eigenbases } \\
\hline 4.277 & \begin{tabular}{llll|}
2.715 & 2.715 & 2.715 \\
\end{tabular} & $\begin{array}{|ll|}2.138 & 2.138 \\
\end{array}$ & 1.905 & )$^{\top}$ \\
\hline 0.894 & $-0.200-0.200-0.200$ & $\begin{array}{|ll|}0.447 & 0.447 \\
\end{array}$ & -0.672 & $10)^{\top}$ \\
\hline 0 & \multicolumn{4}{|c|}{ 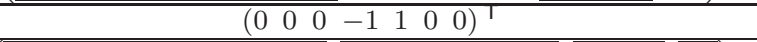 } \\
\hline-0.614 & $\begin{array}{llll}-0.270 & -0.270 & -0.270 \\
\end{array}$ & $-0.307-0.307$ & 1.321 & $1)^{\top}$ \\
\hline-1 & \multicolumn{4}{|c|}{$\left.\begin{array}{llllllll} & \left(\begin{array}{lllllll}-1 & 0 & 1 & 0 & 0 & 0 & 0\end{array}\right)^{\top} \\
& (-1 & 1 & 0 & 0 & 0 & 0 & 0\end{array}\right)^{\mathrm{T}}$} \\
\hline-2.556 & $\begin{array}{|lll|}0.756 & 0.756 & 0.756 \\
\end{array}$ & $-1.278-1.278$ & -0.887 & $1)^{\top}$ \\
\hline
\end{tabular}

\begin{tabular}{|c|c|c|c|c|c|c|}
\hline Eigenvalues of $\mathcal{S}$ & \multicolumn{6}{|c|}{ Eigenbases } \\
\hline 4.123 & $-1.281-1.281-1.28$ & -0.360 & -0.360 & - & & 1 ] ${ }^{\mathrm{T}}$ \\
\hline 2 & $\begin{array}{lll}1 & 1 & 1 \\
\end{array}$ & 33 & $3)^{\top}$ & & & \\
\hline 1 & \multicolumn{6}{|c|}{$\left(\begin{array}{lllllll}0 & 0 & 0 & -1 & 1 & 0 & 0\end{array}\right)$} \\
\hline-1 & \multicolumn{6}{|c|}{ 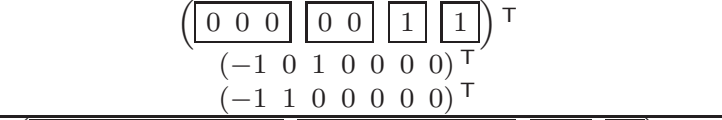 } \\
\hline-4.123 & $\begin{array}{lll}0.781 & 0.781 & 0.781 \\
\end{array}$ & $-1.390-$ & 1.390 & -1 & 1 & $\mathrm{~T}$ \\
\hline
\end{tabular}

The eigenspaces of $\mathbf{A}, \mathbf{L}, \mathbf{Q}, \overline{\mathbf{A}}$ and $\mathcal{S}$ of the graph in Figure 2.1. 
agent connected to some follower agents of $G$. If the leader happens to be adjacent to all the vertices (followers) of $G$, then the system graph would be $G_{\triangleright}$, the cone of $G$. Indeed, we shall be considering this scenario below.

Let us first consider a networked dynamical system of $n$ agents with no leaders. The dynamics $\dot{x}_{i}$ of the state $x_{i}$ of the $i^{\text {th }}$ agent is taken to depend on those of its neighbours. These dynamics are usually of the following form:

$$
\begin{array}{r}
\dot{x}_{i}(t)=\gamma_{A}\left(\sum_{\{i, j\} \in \mathcal{E}(G)} x_{j}(t)\right)+\gamma_{D}\left(\sum_{\{i, j\} \in \mathcal{E}(G)} x_{i}(t)\right)+\gamma_{I} x_{i}(t)+\gamma_{J}\left(\sum_{j=1}^{n} x_{j}(t)\right) \\
i \in\{1, \ldots, n\}
\end{array}
$$

where $\gamma_{A}$ is a nonzero constant and $\gamma_{D}, \gamma_{I}$ and $\gamma_{J}$ are arbitrary constants (which may possibly be zero). If we denote the vector $\left(\begin{array}{llll}x_{1} & x_{2} & \cdots & x_{n}\end{array}\right)^{\top}$ by $\mathbf{x}(t)$ and the vector $\left(\begin{array}{llll}\dot{x}_{1} & \dot{x}_{2} & \cdots & \dot{x}_{n}\end{array}\right)^{\top}$ by $\dot{\mathbf{x}}(t)$, we can write down the $n$ dynamical equations in (3.1) as the one vector equation:

$$
\dot{\mathbf{x}}(t)=\left(\gamma_{A} \mathbf{A}+\gamma_{D} \mathbf{D}+\gamma_{I} \mathbf{I}+\gamma_{J} \mathbf{J}\right) \mathbf{x}(t)=\mathbf{U} \mathbf{x}(t),
$$

where $\mathbf{U}$ is the universal adjacency matrix of the graph $G$ akin to (2.1).

Let us now add one leader agent adjacent to every follower in $G$ to the networked dynamical system of $n$ followers, so that we form the system graph $G_{\triangleright}$ as in Figure 3.1. This leader does not follow the dynamics described in (3.2), but it introduces an arbitrary input $u(t)$ to each of the followers instead. Thus, the system now satisfies the differential equation

$$
\dot{\mathbf{x}}(t)=\mathbf{U} \mathbf{x}(t)+\mathbf{j} u(t)
$$

If, starting from any initial state, it is possible for the leader agent to transfer the followers in finite time to a state chosen arbitrarily in advance, then the underlying networked dynamical system is said to be controllable. If the networked dynamical system in (3.3) is controllable, then $(\mathbf{U}, \mathbf{j})$ is referred to as a controllable pair $[2$, Definition 6.1].

4. U-Controllable graphs. In view of what was just presented in the previous section, we define a $\mathbf{U}$-controllable graph as follows:

Definition 4.1. A simple graph $G$ on $n$ vertices having an associated universal adjacency matrix $\mathbf{U}$ as in (2.1) is said to be $\mathbf{U}$-controllable if $(\mathbf{U}, \mathbf{j})$ is a controllable pair, that is, if the system (3.3) with the same universal adjacency matrix $\mathbf{U}$ as that of $G$ is controllable. 

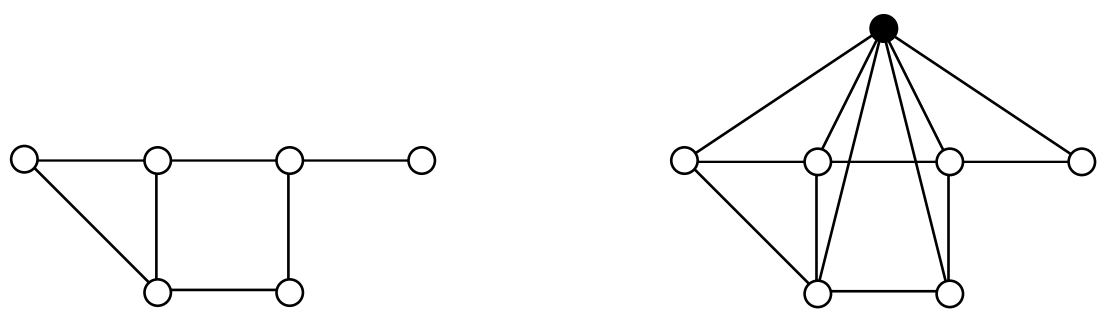

FIG. 3.1. A follower graph (left), and its cone graph (right).

The terminology $\mathbf{U}(G)$-controllable shall be used instead of the term "U-controllable" whenever an emphasis on the graph $G$ in question is deemed necessary.

In the rest of this section, we shall determine necessary and sufficient conditions for a graph to be $\mathbf{U}$-controllable. To this end, we first combine several results that were presented in [6] in the theorem below.

TheOREM 4.2. [6] Let $\mathbf{F}$ be an $n \times n$ real and symmetric matrix representing some graph $G$, let $\mathbf{r}$ be any $n \times 1$ vector and let

$$
\mathbf{S}=\left(\begin{array}{cc}
\mathbf{F} & \mathbf{r} \\
\mathbf{r}^{\top} & c
\end{array}\right)
$$

for some arbitrary real constant c. Then the following five statements are equivalent:

(i) $(\mathbf{F}, \mathbf{r})$ is a controllable pair.

(ii) The controllability matrix $\left(\begin{array}{llllll}\mathbf{r} & \mathbf{F r} & \mathbf{F}^{2} \mathbf{r} & \cdots & \mathbf{F}^{n-1} \mathbf{r}\end{array}\right)$ has full rank $n$.

(iii) No eigenvector of $\mathbf{F}$ is orthogonal to $\mathbf{r}$.

(iv) No eigenvector of $\mathbf{S}$ has its last entry equal to zero.

(v) The matrices $\mathbf{F}$ and $\mathbf{S}$ have no common eigenvalues.

We require $(\mathbf{U}, \mathbf{j})$ to be a controllable pair. The matrix $\mathbf{S}$ of Theorem 4.2 is the system matrix of $G$, which, for the system (3.3) being considered, is

$$
\mathbf{U}_{S}=\left(\begin{array}{ll}
\mathbf{U} & \mathbf{j} \\
\mathbf{j}^{\top} & c
\end{array}\right),
$$

where $c$ is an arbitrary scalar. By Theorem 4.2 (iii), a graph $G$ is $\mathbf{U}$-controllable if and only if no eigenvector of $\mathbf{U}$ is orthogonal to $\mathbf{j}$. In other words, $G$ is $\mathbf{U}$-controllable if and only if $\mathbf{U}$ has only main eigenvectors.

We remark that if $\lambda$ is an eigenvalue of $\mathbf{U}$ having two linearly independent main eigenvectors $\mathbf{x}_{1}$ and $\mathbf{x}_{2}$, then the vector $\mathbf{x}=\left(\mathbf{j}^{\top} \mathbf{x}_{2}\right) \mathbf{x}_{1}-\left(\mathbf{j}^{\top} \mathbf{x}_{1}\right) \mathbf{x}_{2}$ is a non-main eigenvector of $\lambda$, and thus, the graph would not be $\mathbf{U}$-controllable. Hence, for a graph to be $\mathbf{U}$-controllable, all the eigenvalues of $\mathbf{U}$ must be simple and main. 
We thus have the following five necessary and sufficient conditions for a graph to be $\mathbf{U}$-controllable.

THEOREM 4.3. Let $\mathbf{U}$ be the $n \times n$ universal adjacency matrix as in (2.1) associated with a simple graph $G$, and let $\mathbf{U}_{S}$ be as in (4.1). Then the following six statements are equivalent:

(i) $G$ is a $\mathbf{U}$-controllable graph.

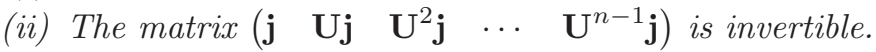

(iii) $\mathbf{U}$ has only main eigenvectors.

(iv) All the eigenvalues in the spectrum of $\mathbf{U}$ are simple and main.

(v) No eigenvector of $\mathbf{U}_{S}$ has its last entry equal to zero.

(vi) The matrices $\mathbf{U}$ and $\mathbf{U}_{S}$ have no common eigenvalues.

4.1. The universal adjacency matrix of the cone graph. Let us consider the matrix $\mathbf{U}_{\triangleright}$, the universal adjacency matrix of $G_{\triangleright}$ with the same parameters as those used for the universal adjacency matrix of $G$. We now pose the question: for which class of graphs will considering $\mathbf{U}_{\triangleright}$ be sufficient to determine whether $G$ is U-controllable?

To answer this question, we expand $\mathbf{U}_{\triangleright}=\gamma_{A} \mathbf{A}_{\triangleright}+\gamma_{D} \mathbf{D}_{\triangleright}+\gamma_{I} \mathbf{I}_{\triangleright}+\gamma_{J} \mathbf{J}_{\triangleright}$, where

$$
\mathbf{A}_{\triangleright}=\left(\begin{array}{cc}
\mathbf{A} & \mathbf{j} \\
\mathbf{j}^{\top} & 0
\end{array}\right), \quad \mathbf{D}_{\triangleright}=\left(\begin{array}{cc}
\mathbf{D}+\mathbf{I} & \mathbf{0} \\
\mathbf{0}^{\top} & n
\end{array}\right), \quad \mathbf{I}_{\triangleright}=\left(\begin{array}{cc}
\mathbf{I} & \mathbf{0} \\
\mathbf{0}^{\top} & 1
\end{array}\right), \quad \mathbf{J}_{\triangleright}=\left(\begin{array}{cc}
\mathbf{J} & \mathbf{j} \\
\mathbf{j}^{\top} & 1
\end{array}\right) .
$$

Thus,

$$
\begin{aligned}
\mathbf{U}_{\triangleright} & =\gamma_{A}\left(\begin{array}{ll}
\mathbf{A} & \mathbf{j} \\
\mathbf{j}^{\top} & 0
\end{array}\right)+\gamma_{D}\left(\begin{array}{cc}
\mathbf{D}+\mathbf{I} & \mathbf{0} \\
\mathbf{0}^{\top} & n
\end{array}\right)+\gamma_{I}\left(\begin{array}{cc}
\mathbf{I} & \mathbf{0} \\
\mathbf{0}^{\top} & 1
\end{array}\right)+\gamma_{J}\left(\begin{array}{cc}
\mathbf{J} & \mathbf{j} \\
\mathbf{j}^{\top} & 1
\end{array}\right) \\
& =\left(\begin{array}{cc}
\gamma_{A} \mathbf{A} & \gamma_{A} \mathbf{j} \\
\gamma_{A} \mathbf{j}^{\top} & 0
\end{array}\right)+\left(\begin{array}{cc}
\gamma_{D} \mathbf{D}+\gamma_{D} \mathbf{I} & \mathbf{0} \\
\mathbf{0}^{\top} & \gamma_{D} n
\end{array}\right)+\left(\begin{array}{cc}
\gamma_{I} \mathbf{I} & \mathbf{0} \\
\mathbf{0}^{\top} & \gamma_{I}
\end{array}\right)+\left(\begin{array}{cc}
\gamma_{J} \mathbf{J} & \gamma_{J} \mathbf{j} \\
\gamma_{J} \mathbf{j}^{\top} & \gamma_{J}
\end{array}\right) \\
& =\left(\begin{array}{cc}
\gamma_{A} \mathbf{A}+\gamma_{D} \mathbf{D}+\gamma_{D} \mathbf{I}+\gamma_{I} \mathbf{I}+\gamma_{J} \mathbf{J} & \gamma_{A} \mathbf{j}+\gamma_{J} \mathbf{j} \\
\gamma_{A} \mathbf{j}^{\top}+\gamma_{J} \mathbf{j}^{\top} & \gamma_{D} n+\gamma_{I}+\gamma_{J}
\end{array}\right) \\
& =\left(\begin{array}{cc}
\mathbf{U}+\gamma_{D} \mathbf{I} & \left(\gamma_{A}+\gamma_{J}\right) \mathbf{j} \\
\left(\gamma_{A}+\gamma_{J}\right) \mathbf{j}^{\top} & \gamma_{D} n+\gamma_{I}+\gamma_{J}
\end{array}\right) .
\end{aligned}
$$

It is important to stress that, in general, the matrix $\mathbf{U}_{\triangleright}$ in (4.2) is different from the system matrix $\mathbf{U}_{S}$ in (4.1).

We now present the following result that gives another characterization of $\mathbf{U}_{-}$ controllable graphs, but only for those whose universal adjacency matrix satisfies the relation $\gamma_{A}+\gamma_{J} \neq 0$. 
THEOREM 4.4. Let $\mathbf{U}$ be the universal adjacency matrix of a graph $G$ whose parameters satisfy the relation $\gamma_{A}+\gamma_{J} \neq 0$ and let $\mathbf{U}_{\triangleright}$ be the universal adjacency matrix of $G_{\triangleright}$ with the same parameters as those of $\mathbf{U}$. Then $G$ is $\mathbf{U}$-controllable if and only if, whenever $\lambda$ is an eigenvalue of $\mathbf{U},\left(\lambda+\gamma_{D}\right)$ is not an eigenvalue of $\mathbf{U}_{\triangleright}$.

Proof. We prove the contrapositive statement. So suppose $G$ is not $\mathbf{U}$-controllable. By Theorem 4.3 (iii), we can find an eigenvalue $\lambda$ of $\mathbf{U}$ with a non-main eigenvector $\mathbf{x}$. Let $k=\gamma_{D} n+\gamma_{I}+\gamma_{J}$. Then

$$
\mathbf{U}_{\triangleright}\left(\begin{array}{c}
\mathbf{x} \\
0
\end{array}\right)=\left(\begin{array}{cc}
\mathbf{U}+\gamma_{D} \mathbf{I} & \left(\gamma_{A}+\gamma_{J}\right) \mathbf{j} \\
\left(\gamma_{A}+\gamma_{J}\right) \mathbf{j}^{\top} & k
\end{array}\right)\left(\begin{array}{c}
\mathbf{x} \\
0
\end{array}\right)=\left(\begin{array}{c}
\mathbf{U} \mathbf{x}+\gamma_{D} \mathbf{x} \\
\left(\gamma_{A}+\gamma_{J}\right) \mathbf{j}^{\top} \mathbf{x}
\end{array}\right)=\left(\lambda+\gamma_{D}\right)\left(\begin{array}{c}
\mathbf{x} \\
0
\end{array}\right)
$$

and hence, $\left(\lambda+\gamma_{D}\right)$ is an eigenvalue of $\mathbf{U}_{\triangleright}$.

Conversely, suppose $\lambda$ is an eigenvalue of $\mathbf{U}$ with associated eigenvector $\mathbf{x}$ and $\left(\lambda+\gamma_{D}\right)$ is an eigenvalue of $\mathbf{U}_{\triangleright}$ with associated eigenvector $\left(\mathbf{y}^{\top} c\right)^{\top}$. Then

$$
\begin{aligned}
\mathbf{U}_{\triangleright}\left(\begin{array}{l}
\mathbf{y} \\
c
\end{array}\right) & =\left(\begin{array}{cc}
\mathbf{U}+\gamma_{D} \mathbf{I} & \left(\gamma_{A}+\gamma_{J}\right) \mathbf{j} \\
\left(\gamma_{A}+\gamma_{J}\right) \mathbf{j}^{\top} & k
\end{array}\right)\left(\begin{array}{l}
\mathbf{y} \\
c
\end{array}\right) \\
& =\left(\begin{array}{c}
\mathbf{U} \mathbf{y}+\gamma_{D} \mathbf{y}+\left(\gamma_{A}+\gamma_{J}\right) c \mathbf{j} \\
\left(\gamma_{A}+\gamma_{J}\right) \mathbf{j}^{\top} \mathbf{y}+c k
\end{array}\right)=\left(\lambda+\gamma_{D}\right)\left(\begin{array}{l}
\mathbf{y} \\
c
\end{array}\right) .
\end{aligned}
$$

Hence, we have:

$$
\begin{aligned}
\mathbf{U y}+\left(\gamma_{A}+\gamma_{J}\right) c \mathbf{j} & =\lambda \mathbf{y} \\
\left(\gamma_{A}+\gamma_{J}\right) \mathbf{j}^{\top} \mathbf{y}+c k & =c\left(\lambda+\gamma_{D}\right)
\end{aligned}
$$

If we premultiply (4.3) by $\mathbf{x}^{\top}$, we obtain

$$
\begin{aligned}
\mathbf{x}^{\top} \mathbf{U} \mathbf{y}+ & \left(\gamma_{A}+\gamma_{J}\right) c \mathbf{x}^{\top} \mathbf{j}=\lambda \mathbf{x}^{\top} \mathbf{y} \\
\left(\gamma_{A}+\gamma_{J}\right) c \mathbf{j}^{\top} \mathbf{x} & =0 .
\end{aligned}
$$

But $\gamma_{A}+\gamma_{J} \neq 0$, so either $\mathbf{j}^{\top} \mathbf{x}=0$ or $c=0$.

If $\mathbf{j}^{\top} \mathbf{x}=0$, then we have found a non-main eigenvector of $\mathbf{U}$, and consequently, $G$ is not $\mathbf{U}$-controllable by Theorem 4.3 (iii).

If $c=0$, then (4.3) becomes $\mathbf{U y}=\lambda \mathbf{y}$. Moreover, (4.4) becomes $\left(\gamma_{A}+\gamma_{J}\right) \mathbf{j}^{\top} \mathbf{y}=0$, from which $\mathbf{j}^{\top} \mathbf{y}=0$. Thus, $\mathbf{y}$ is a non-main eigenvector of $\mathbf{U}$, so by Theorem 4.3 (iii) again, $G$ is not $\mathbf{U}$-controllable.

Note that Theorem 4.4 holds for the most commonly used matrices, but not, for instance, for $\overline{\mathbf{A}}$. So the theorem cannot be applied to test whether a graph is $\overline{\mathbf{A}}-$ controllable. 
4.2. A-Controllable and $\mathbf{Q}$-controllable graphs. A controllable graph $G$, as defined in [4], is a simple graph $G$ with the spectrum of $\mathbf{A}$ consisting entirely of distinct and main eigenvalues. Similarly, a Q-controllable graph [5] is a simple graph $G$ whose signless Laplacian matrix $\mathbf{Q}$ has eigenvalues that are simple and main. Thus, by Theorem 4.3 (i) and (iv), these two aforementioned classes of graphs are represented in our work by $\mathbf{A}$-controllable graphs and $\mathbf{Q}-$ controllable graphs respectively.

We have the following two corollaries to Theorem 4.4, which were originally proved in [6] (Theorems 4.3 and 4.4) in a different manner.

Corollary 4.5. [6] A graph $G$ is $\mathbf{A}$-controllable if and only if the adjacency matrices of $G$ and $G_{\triangleright}$ have no common eigenvalues.

Proof. Apply Theorem 4.4 with $\gamma_{D}=0$.

Corollary 4.6. [6] A graph $G$ is $\mathbf{Q}$-controllable if and only if $(\lambda+1)$ is not an eigenvalue of the signless Laplacian matrix of $G_{\triangleright}$ whenever $\lambda$ is an eigenvalue of $\mathbf{Q}$.

Proof. Apply Theorem 4.4 with $\gamma_{D}=1$.

As mentioned earlier, a relation between the number of main eigenvalues of the matrices $\mathbf{A}$ and $\mathbf{Q}$ associated with the same graph $G$ is not known. Indeed, Figure 4.1 shows two graphs, the first of which is $\mathbf{A}$-controllable but not $\mathbf{Q}-$-controllable, and the second is $\mathbf{Q}$-controllable but not $\mathbf{A}$-controllable. The graph in Figure 2.1 is neither $\mathbf{A}$-controllable nor $\mathbf{Q}-$-controllable, but the matrices $\mathbf{A}$ and $\mathbf{Q}$ associated with it have the same number (four) of main eigenvalues.
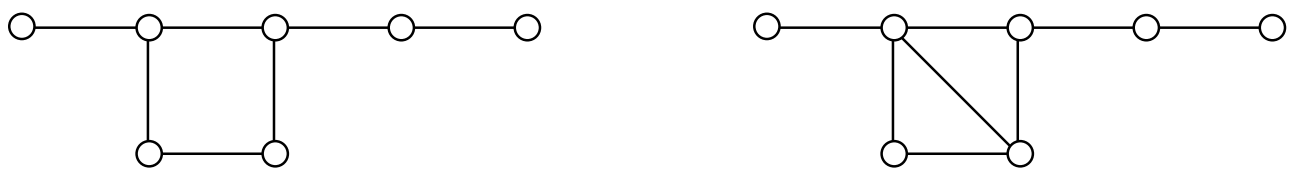

FIG. 4.1. An $\mathbf{A}$-controllable graph which is not $\mathbf{Q}$-controllable (left), and a $\mathbf{Q}$-controllable graph which is not $\mathbf{A}$-controllable (right).

5. The automorphism group of $\mathbf{U}$-controllable graphs. Henceforth, we make use of Theorem 4.3 to test whether a graph $G$ is $\mathbf{U}$-controllable.

It is known that the automorphism group of $\mathbf{A}$-controllable graphs is trivial $[4,7]$. This result can be shown to be true for $\mathbf{U}$-controllable graphs as well.

THEOREM 5.1. If $G$ is a $\mathbf{U}$-controllable graph for a universal adjacency matrix $\mathbf{U}$, then $G$ is asymmetric.

Proof. By Theorem 4.3 (iv), all the eigenvalues of $\mathbf{U}$ are distinct and main. 
Thus, by Theorem 2.4, $G$ has at least $n$ orbits, and therefore must have a trivial automorphism group.

We note that the converse to Theorem 5.1 is false by considering regular asymmetric graphs, of which the Frucht graph, depicted in Figure 5.1, is the smallest example among graphs of degree three.

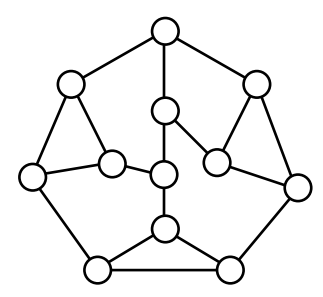

FIG. 5.1. The Frucht graph is an asymmetric graph which is not $\mathbf{U}$-controllable.

THEOREM 5.2. If $G$ is an asymmetric regular graph on at least two vertices, then $G$ is not $\mathbf{U}$-controllable whatever the parameters of the universal adjacency matrix $\mathbf{U}$ associated with $G$ are.

Proof. Let $G$ have degree $\rho$, so that $\mathbf{A} \mathbf{j}=\rho \mathbf{j}$ and $\mathbf{D}=\rho \mathbf{I}$. Consider $\mathbf{U} \mathbf{j}$ :

$$
\mathbf{U} \mathbf{j}=\gamma_{A} \mathbf{A} \mathbf{j}+\gamma_{D}(\rho \mathbf{I}) \mathbf{j}+\gamma_{I} \mathbf{I} \mathbf{j}+\gamma_{J} \mathbf{J} \mathbf{j}=\gamma_{A} \rho \mathbf{j}+\left(\rho \gamma_{D}+\gamma_{I}\right) \mathbf{j}+n \gamma_{J} \mathbf{j}
$$

Hence, $\lambda=\rho\left(\gamma_{A}+\gamma_{D}\right)+\gamma_{I}+n \gamma_{J}$ is an eigenvalue of $\mathbf{U}$ with associated eigenvector j. Since $\mathbf{U}$ is real and symmetric and $n>1$, it has an orthogonal set of eigenvectors, one of which is $\mathbf{j}$. Thus, there must exist at least one non-main eigenvector of $\mathbf{U}$. Hence, by Theorem 4.3 (iii), $G$ is not $\mathbf{U}$-controllable.

6. The $\gamma$-Laplacian matrix. In Section 7, we show that there exist nonregular asymmetric graphs that are not $\mathbf{U}$-controllable for any universal adjacency matrix $\mathbf{U}$. To arrive at this result, we introduce a simpler form of a universal adjacency matrix.

For a scalar $\gamma$, the matrix $\mathbf{A}+\gamma \mathbf{D}$ associated with a graph $G$, denoted by $\mathcal{L}(\gamma)$, will be called the $\gamma$-Laplacian matrix of $G$. Note that the matrices $\mathcal{L}(0), \mathcal{L}(1)$ and $\mathcal{L}(-1)$ are the adjacency matrix $\mathbf{A}$, the signless Laplacian matrix $\mathbf{Q}$ and the negative Laplacian matrix $-\mathbf{L}$ respectively. Moreover, a $\gamma$-Laplacian matrix is a universal adjacency matrix with parameters $\gamma_{A}=1, \gamma_{D}=\gamma$ and $\gamma_{I}=\gamma_{J}=0$.

In Theorem 6.4, we shall show that a $\mathcal{L}(\gamma)$-controllable graph is also $\mathbf{U}$-controllable for appropriate parameters of $\mathbf{U}$. First, we require the following two lemmas.

LEMMA 6.1. The graph $G$ is $\mathbf{U}$-controllable for some universal adjacency matrix $\mathbf{U}$ if and only if $G$ is $(k \mathbf{U})$-controllable for some nonzero scalar $k$. 
Proof. The matrices $\mathbf{U}$ and $k \mathbf{U}$ have the same eigenvectors. Since, by Theorem 4.3 (iii), $G$ is $\mathbf{U}$-controllable if and only if $\mathbf{U}$ has only main eigenvectors, the result follows. $\square$

LEMMA 6.2. The graph $G$ is $\mathbf{U}$-controllable for some universal adjacency matrix $\mathbf{U}$ if and only if $G$ is $(\mathbf{U}+\alpha \mathbf{I}+\beta \mathbf{J})$-controllable for any scalars $\alpha$ and $\beta$.

Proof. We are going to prove the contrapositive statement. So suppose $G$ is not $\mathbf{V}$-controllable, where $\mathbf{V}=\mathbf{U}+\alpha \mathbf{I}+\beta \mathbf{J}$. By Theorem 4.3 (iii), there exists an eigenvalue $\lambda$ of $\mathbf{V}$ with an associated non-main eigenvector $\mathbf{x}$. But $\mathbf{V} \mathbf{x}=\lambda \mathbf{x}$ if and only if $\mathbf{U x}=(\lambda-\alpha) \mathbf{x}$, which means that $\mathbf{x}$ is also an eigenvector of $\mathbf{U}$. It follows that $G$ is not $\mathbf{V}$-controllable if and only if it is not $\mathbf{U}$-controllable.

The following corollary is immediate from Lemma 6.2.

Corollary 6.3. A graph is $\mathbf{A}$-controllable if and only if it is $\mathbf{A}$-controllable if and only if it is $\mathcal{S}$-controllable.

We remark that if the universal adjacency matrix of a graph $G$ is $\overline{\mathbf{A}}$, then the system matrix $\overline{\mathbf{A}}_{S}$ is

$$
\left(\begin{array}{ll}
\overline{\mathbf{A}} & \mathbf{j} \\
\mathbf{j} & c
\end{array}\right)
$$

Observe that if the universal adjacency matrix of $\bar{G}$, the complement of $G$, is $\mathbf{A}(\bar{G})$, then the system matrix of $\bar{G}$ is (6.1) as well. Thus, $G$ is $\overline{\mathbf{A}}$-controllable if and only if $\bar{G}$ is $\mathbf{A}(\bar{G})$-controllable by Theorem $4.3(\mathrm{v})$. Therefore, by Corollary $6.3, G$ is A-controllable if and only if $\bar{G}$ is $\mathbf{A}(\bar{G})$-controllable, a result that was noted in [7].

The next result allows us to considerably simplify our work related to $\mathbf{U}$-controllable graphs.

TheOREM 6.4. A graph $G$ is $\mathcal{L}(\gamma)$-controllable for some parameter $\gamma$ if and only if $G$ is $\mathbf{U}$-controllable for parameters satisfying $\gamma=\frac{\gamma_{D}}{\gamma_{A}}$.

Proof. Let $\mathcal{L}(\gamma)=\mathbf{A}+\gamma$ D. By Lemma $6.1, G$ is $\mathcal{L}(\gamma)$-controllable if and only if $G$ is $\left(\gamma_{A} \mathbf{A}+\gamma_{A} \gamma \mathbf{D}\right)$-controllable for some nonzero scalar $\gamma_{A}$. By Lemma 6.2, $G$ is $\left(\gamma_{A} \mathbf{A}+\gamma_{A} \gamma \mathbf{D}\right)$-controllable if and only if $G$ is $\left(\gamma_{A} \mathbf{A}+\gamma_{A} \gamma \mathbf{D}+\gamma_{I} \mathbf{I}+\gamma_{J} \mathbf{J}\right)$-controllable, where $\gamma_{I}$ and $\gamma_{J}$ are arbitrary scalars. Hence, the result follows.

Thus, by Theorem 6.4, it suffices to consider only the simpler $\gamma$-Laplacian matrices of the form $\mathbf{A}+\gamma \mathbf{D}$ to test for $\mathbf{U}$-controllable graphs with $\gamma=\frac{\gamma_{D}}{\gamma_{A}}$ and arbitrary values of $\gamma_{I}$ and $\gamma_{J}$.

Recall that $\mathcal{L}(-1)=-\mathbf{L}$. The only $\mathbf{L}$-controllable graph is $K_{1}$, the trivial graph on one vertex, as 0 is the only main eigenvalue of the Laplacian matrix $\mathbf{L}$. 
Furthermore, $K_{1}$ is $\mathcal{L}(\gamma)$-controllable for all scalars $\gamma$. By applying Theorem 6.4 , we obtain that $K_{1}$ is the only graph that is $\mathbf{U}$-controllable for every possible universal adjacency matrix $\mathbf{U}$.

7. Non-regular asymmetric graphs. By Theorems 5.1 and 5.2, any Ucontrollable graph with $n>1$ must be asymmetric and non-regular. Can it be the case that any non-regular asymmetric graph is $\mathbf{U}$-controllable for some universal adjacency matrix $\mathbf{U}$ ? It transpires that the answer is in the negative.

THEOREM 7.1. There exist non-regular asymmetric graphs that are not $\mathbf{U}-$ controllable whatever the universal adjacency matrix $\mathbf{U}$ is.

Proof. Consider the non-regular, asymmetric graph $G$ in Figure 7.1 and consider the matrix $(\mathbf{A}+\gamma \mathbf{D})$ associated with $G$ for some arbitrary constant $\gamma$ :

$$
\mathbf{A}+\gamma \mathbf{D}=\left(\begin{array}{ccccccc}
\gamma & 1 & 0 & 0 & 0 & 0 & 0 \\
1 & 3 \gamma & 1 & 1 & 0 & 0 & 0 \\
0 & 1 & 2 \gamma & 1 & 0 & 0 & 0 \\
0 & 1 & 1 & 3 \gamma & 1 & 0 & 0 \\
0 & 0 & 0 & 1 & 2 \gamma & 1 & 0 \\
0 & 0 & 0 & 0 & 1 & 2 \gamma & 1 \\
0 & 0 & 0 & 0 & 0 & 1 & \gamma
\end{array}\right)
$$

Since

$$
\left(\begin{array}{ccccccc}
\gamma & 1 & 0 & 0 & 0 & 0 & 0 \\
1 & 3 \gamma & 1 & 1 & 0 & 0 & 0 \\
0 & 1 & 2 \gamma & 1 & 0 & 0 & 0 \\
0 & 1 & 1 & 3 \gamma & 1 & 0 & 0 \\
0 & 0 & 0 & 1 & 2 \gamma & 1 & 0 \\
0 & 0 & 0 & 0 & 1 & 2 \gamma & 1 \\
0 & 0 & 0 & 0 & 0 & 1 & \gamma
\end{array}\right)\left(\begin{array}{c}
0 \\
0 \\
-1 \\
1 \\
-\gamma \\
\gamma-1 \\
1
\end{array}\right)=\left(\begin{array}{c}
0 \\
0 \\
1-2 \gamma \\
2 \gamma-1 \\
\gamma-2 \gamma^{2} \\
2 \gamma^{2}-3 \gamma+1 \\
2 \gamma-1
\end{array}\right)=(2 \gamma-1)\left(\begin{array}{c}
0 \\
0 \\
-1 \\
1 \\
-\gamma \\
\gamma-1 \\
1
\end{array}\right)
$$

the matrix $\mathbf{A}+\gamma \mathbf{D}$ has an eigenvalue $\lambda_{\gamma}=(1-2 \gamma)$ with associated eigenvector $\mathbf{x}_{\gamma}=\left(\begin{array}{lllllll}0 & 0 & -1 & 1 & -\gamma & \gamma-1 & 1\end{array}\right)^{\top}$. Since $\mathbf{x}_{\gamma}$ is a non-main eigenvector, the graph $G$ is not $\mathcal{L}(\gamma)$-controllable by Theorem 4.3 (iii). By Theorem 6.4, this means that $G$ is not $\mathbf{U}$-controllable for any universal adjacency matrix $\mathbf{U}$.

Hence, for $n>1$, the $\mathbf{U}$-controllable graphs form a proper subfamily of the family of all asymmetric, non-regular graphs. 


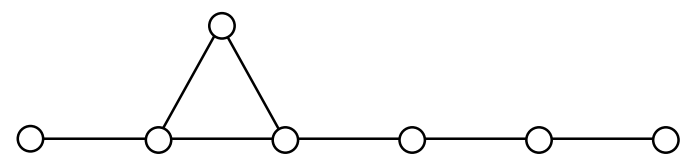

FIG. 7.1. A non-regular, asymmetric graph that is not $\mathbf{U}$-controllable for any universal adjacency matrix $\mathbf{U}$.

8. Conclusion. Graphs on at least two vertices that are $\mathbf{A}$-controllable or $\mathbf{Q}-$ controllable graphs have been considered in the literature and are both shown to be asymmetric and non-regular. The converse does not hold for either class of graphs.

One may pose the question whether all non-regular asymmetric graphs are $\mathbf{U}-$ controllable for a more general universal adjacency matrix $\mathbf{U}$ of the form (2.1). We have shown that $\mathbf{U}$-controllable graphs are non-regular and asymmetric for any parameters $\gamma_{A}, \gamma_{D}, \gamma_{I}$ and $\gamma_{J}$ of $\mathbf{U}$, but there exist non-regular asymmetric graphs that are not $\mathbf{U}$-controllable no matter which parameters are chosen for the matrix $\mathbf{U}$, as shown by Theorem 7.1. It would be interesting to consider other combinatorial and structural properties that would further restrict the family of $\mathbf{U}$-controllable graphs within the family of non-regular, asymmetric graphs.

\section{REFERENCES}

[1] B. Ahmadi, F. Alinaghipour, S.M. Fallat, Y.-Z. Fan, K. Meagher, and S. Nasserasr. The minimum rank of universal adjacency matrices. Linear Algebra and its Applications, 437(8):2064-2076, 2012.

[2] C.-T. Chen. Linear System Theory and Design. Oxford University Press, Inc., New York, 1998.

[3] D. Cvetković and P.W. Fowler. A group-theoretical bound for the number of main eigenvalues of a graph. J. Chem. Inf. Comput. Sci., 39:638-641, 1999.

[4] D. Cvetković, P. Rowlinson, Z. Stanić, and M.G. Yoon. Controllable graphs. Bulletin, Classe des Sciences Mathématiques et Naturelles, Sciences Mathématiques, CXLIII(36):81-88, 2011.

[5] D. Cvetković, P. Rowlinson, Z. Stanić, and M.G. Yoon. Controllable graphs with least eigenvalue at least -2. Applicable Analysis and Discrete Mathematics, 5:165-175, 2011.

[6] A. Farrugia and I. Sciriha. Controllability of undirected graphs. Linear Algebra and its Applications, 454:138-157, 2014.

[7] C. Godsil. Controllable subsets in graphs. Annals of Combinatorics, 16(4):733-744, 2012.

[8] W.H. Haemers and G.R. Omidi. Universal adjacency matrices with two eigenvalues. Linear Algebra and its Applications, 435(10):2520-2529, 2011.

[9] A. Mowshowitz. The group of a graph whose adjacency matrix has all distinct eigenvalues. Proof Techniques in Graph Theory, Academic Press, New York, 109-110, 1969.

[10] M. Petersdorf and H. Sachs. Spektrum und automorphismengruppe eines graphen. Combinatorial Theory and its Applications III, North-Holland, Amsterdam, 891-907, 1969. 